\title{
EVIDENCE BASED REVIEW OF PHYSIOTHERAPY MANAGEMENT OF CEREBRAL PALSY PATIENTS
}

\author{
Raj Sharma *1, Jyoti Sharma ${ }^{2}$, Vikas Bharadwaj ${ }^{3}$. \\ ${ }^{* 1}$ Assistant Professor, Noida International University, Greater Noida, India. \\ ${ }^{2}$ Assistant Professor, Amar Jyoti Institute of Physiotherapy, Karkardooma, India. \\ ${ }^{3}$ Assistant Professor, School of Medical Sciences and Research, Sharda University, India.
}

\section{ABSTRACT}

Cerebral palsy (CP) is a common neuro developmental condition encountered by pediatricians. The condition may present itself in many different clinical spectra. The etiological and risk factors are many and an awareness of the interplay of multiple factors in the causation of CP is crucial. In many cases, the cause of Cerebral palsy may not be apparent. Cerebral palsy is invariably associated with many deficits such as mental retardation, speech and language and oromotor problems. A thorough neuro developmental assessment of the child with Cerebral Palsy should include evaluation of associated deficits so that a comprehensive early intervention program can be planned and executed. Hence, this review is carried out to assess the benefit of physiotherapy management of cerebral palsy patients.

KEY WORDS: Cerebral Palsy, Physiotherapy management, Mental retardation.

Address for correspondence: Dr. Raj Sharma, Assistant Professor, Noida International University, Greater Noida, India. E-Mail: xyjyoti11@gmail.com

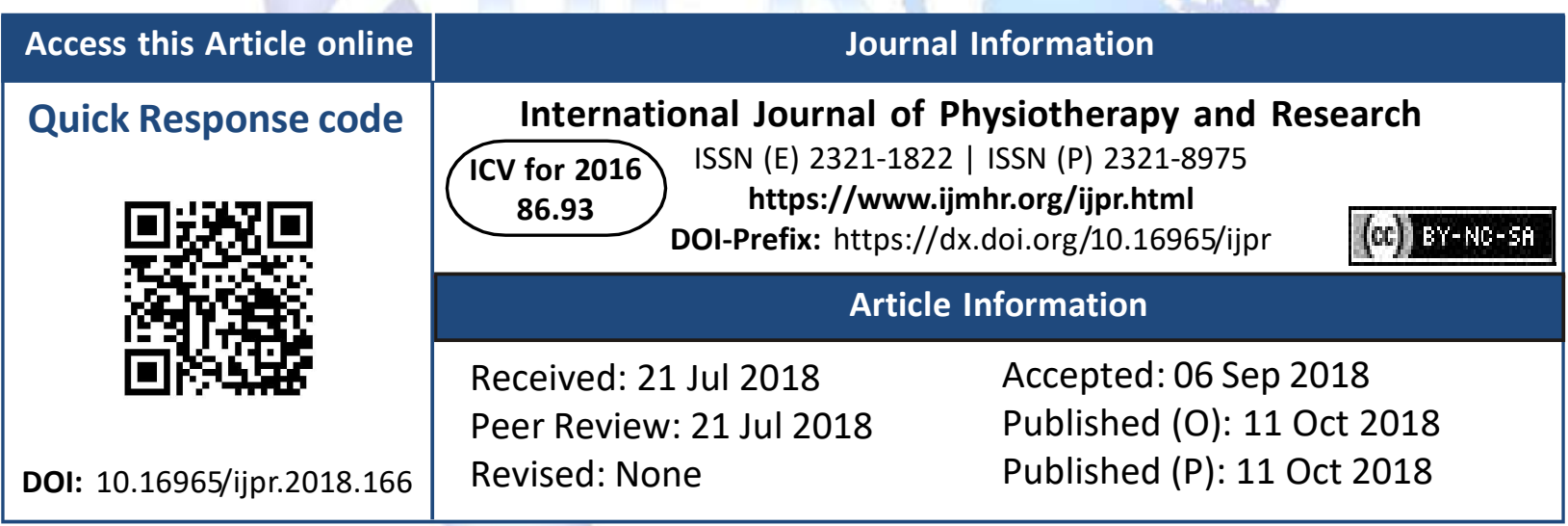

\section{INTRODUCTION}

Cerebral palsy is the most common activity limiting disability in children and adolescents with approximately 2-3 per 1,000 live births worldwide. The incidence rate for cerebral palsy has remained constant since first case recorded in the 1960's, despite advances in the obstetric care of mothers and infants and improved knowledge of some causative factors. The unchanging incidence rate is largely attributed to the increased survival rate of very pre-term and low birth weight infants through enhanced postnatal medical care [1]. Hence, as a result of improved medical care, pre-term infants are more likely to survive, but are more prone to develop associated co-morbidities such as cerebral palsy.

The severity of cerebral palsy is highly variable but inevitably results in some degree of activity limitation due to motor impairment. The motor impairments associated with cerebral palsy can be classified under the framework of the International Classification of Functioning, Disability and Health (ICF) [2]. This framework was developed as a means to describe and measure health and disability, taking into account the physical and social aspects of disability, at both the individual and population levels. The framework is divided into domains of body (comprising body functions and structure), activities and participation (Figure 1). 
Fig. 1: The interaction of the domains of the International Classification of Functioning, Disability, and Health (ICF) (World Health Organization, 2001) in relation to children with cerebral palsy.

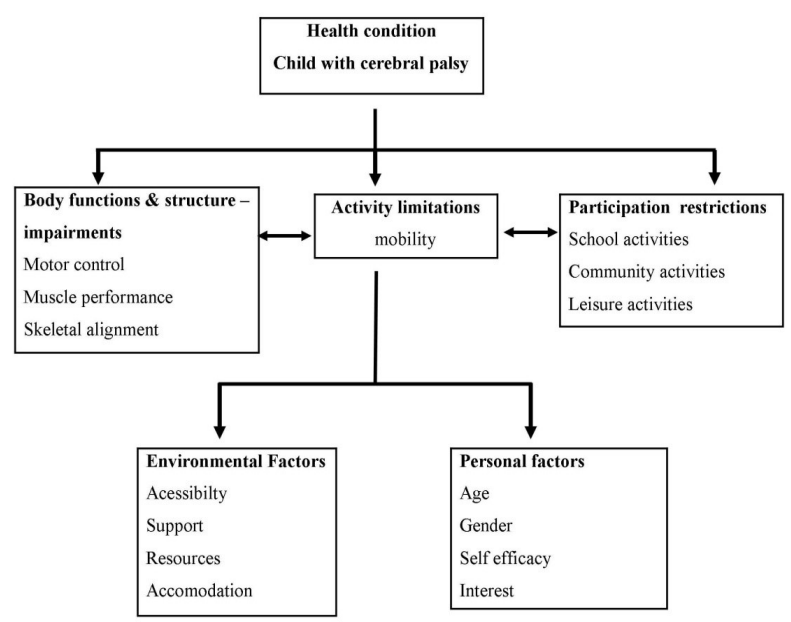

This framework allows physiotherapists to consider the interaction between the child's impairments, their everyday activities and subsequent participation within their community. In this framework, impairments are classified under body functions and structures and describe problems such as impaired or decreased sensation, muscle weakness and contracture. The activity limitations domain describes a child's ability to move and change position in everyday circumstances such as reaching and manipulation, walking, and self-care. Within this domain, children with cerebral palsy may experience difficulties performing age-appropriate tasks or actions [3]. For example, they may be unable to ride a bike or climb on outdoor playground equipment. The domain of participation restrictions identifies how an activity limitation would impact on the child's ability to participate in normally expected life activities [3]. Such activities may include being able to take public transport to go a movie or shopping centre with friends. The domain of environmental and personal factors consider whether such things as a child's age and gender, in addition to accessibility options, support and availability of resources, act as a facilitator or barrier to community participation. Overall, cerebral palsy is a complex condition which spans physical, cognitive and Behavioral domains. However, from the physiotherapist's point of view the Presence of motor impairments, and the subsequent effect that these physical impairments have on a child's activity and participation, are the most relevant for intervention. The challenge for physiotherapists is to assist these children to develop effective motor performance in order that they can be independent in essential activities of everyday life [3]. Children also present a unique challenge in terms of the need to consider the effects of growth and development. In particular, physiotherapists have long recognized the secondary physical impairments of cerebral palsy which become more obvious during periods of growth and the subsequent impact that these changes have on the child's everyday activities [4].

What then is the most effective form of physiotherapy intervention for children with cerebral palsy which will enhance their level of activity and their community participation? In physiotherapy, as in all areas of health care, there is an emphasis on providing services which are based on the best evidence available. Determining what evidence currently exists for physiotherapy intervention for children with cerebral palsy, how strong the evidence is and whether there are any gaps in the available evidence is the focus of this review.

Definition: Cerebral palsy can be broadly defined as a permanent, non-progressive neurological disorder caused by a lesion, or injury, to any part of the developing foetal or infant brain [5]. It is thought that most lesions occur in-utero, however some post-natal events may also lead to cerebral palsy. For example, cerebral palsy is often suspected as a potential outcome where there are pre and peri natal complications of pregnancy. Likewise, where a baby is born extremely premature there is more likelihood that a lesion to the brain may have occurred. It is also known that similar brain lesions may produce different results when they have occurred at different chronological ages in the foetus [6]. For example, a brain lesion in a very preterm infant is more likely to lead to features associated with diplegic cerebral palsy, whereas the same lesion in an infant born at full term is more likely to lead to features associated with hemiplegia, quadriplegia or athetoid cerebral palsy. Cerebral palsy may also be the preliminary diagnosis when there is the presence of abnormal neurological findings in 
the neonatal period, such as inconsistent Apgar scores or delayed developmental milestones.

The definition of cerebral palsy has varied over time, probably as a result of improved understanding of this condition. However, this variation has also led to a lack of consensus in reaching a common definition. As a result, an international workshop of recognized cerebral palsy specialists met in 2007 in an attempt to develop a more inclusive definition.

Cerebral palsy describes a group of disorders of the development of movement and posture, causing activity limitation, that are attributed to non-progressive disturbances that occurred in the developing foetal or infant brain. The motor disorders of CP are often accompanied by disturbances of sensation, cognition, communication, perception, and/or behaviour, and/or by a seizure disorder [7].

This current definition highlights the importance of motor impairment in cerebral palsy while also recognizing the influence of other associated impairments. Although cerebral palsy is acknowledged as being a non-progressive disorder, the impact specifically of musculoskeletal growth on primary physical impairments such as spasticity and weakness, is ongoing throughout childhood and into adulthood [7].

Often therefore the influence of secondary physical impairments such as muscle stiffness, shortening and contracture, impact the child's cognitive, behavioural and communication systems by preventing them from participating in life situations [3].

Incidence: $\mathrm{CP}$ is a common problem, the worldwide incidence being 2 to 2.5 per 1000 live births[8]. When first case of CP was described, the cause was attributed to birth trauma and this view perristed for several decades. Recent advances inneonatal management and obstetric care have not shown a decline in the incidence of CP [9].

On the contrary, with a decline in infant mortality rate, there has actually been an increase in the incidence and severity of CP. The incidence in premature babies is much higher than in term babies. For the vast majority of term infants whodevelop $\mathrm{CP}$, birth asphyxia or obstetric complications cannot be ascribed as the cause [10].

Etiology: Multiple causes have been ascribed to development of CP. Some of which can be linked to a specific time period. Not all causes of $C P$ are well understood. Typical causes of $\mathrm{CP}$ and relationship of these causes with prenatal, perinatal and postnatal occurrences are listed below in table 1.

Table 1: Risk factors associated with cerebral palsy.

\begin{tabular}{|c|c|c|c|}
\hline S. No. & Prental Factors & Perinatal Factors & Post Natal Factors \\
\hline $\mathbf{1}$ & $\begin{array}{c}\text { Maternal infections } \\
\text { Rubella } \\
\text { Herpes simplex } \\
\text { Toxoplasmosis } \\
\text { Cytomegalovirus }\end{array}$ & Asphyxia & Cerebrovascular accident \\
\hline $\mathbf{2}$ & Placental abnormalities & Prolonged labor & Intraventricular hemorrhage \\
\hline $\mathbf{3}$ & Rh incompatibility & Breech birth & $\begin{array}{c}\text { Meningitis } \\
\text { Encephalitis }\end{array}$ \\
\hline $\mathbf{4}$ & Maternal diabetes & Prolapsed cord & Head trauma \\
\hline $\mathbf{5}$ & Toxemia of pregnancy & Compression brain & Near-drowning \\
\hline $\mathbf{6}$ & Brain maldevelopment & Placenta previa & \\
\hline $\mathbf{7}$ & & Prematurity & \\
\hline $\mathbf{8}$ & & Low birth weight & \\
\hline
\end{tabular}

Prenatal cause: When the cause of $\mathrm{CP}$ is known, it is most often related to problems experienced during intrauterine development. A fetus exposed to maternal infections such as rubella, herpes simplex, cytomegalovirus or toxoplasmosis early in gestation can incur damage to the brain is motor centers. If the placenta which provides nutrition and oxygen from the mother does not remain attached to the uterine wall throughout the pregnancy the fetus can be deprived of oxygen and other nutrients. Inflammation of the placenta occur in 50 to 80 percent of premature birth [10] and a risk factor for CP [9].

'Rh factor is found in the red blood cells of $85 \%$ of population. When blood is typed for transfusion or cross matching, both $\mathrm{ABO}$ classification and Rh status are determined. Rh incompatibility occurs when a mother who is Rh-ve delivers a baby who is Rh+ve. The mother becomes sensitive to the baby's blood and begins to make antibodies, if she is not given $\mathrm{Rh}$ immune globulin. The development of maternal antibodies predisposes subsequent pregnancies to $\mathrm{CP}$, high frequency hearing loss, visual problems, and discoloration of the teeth. When the antibody injection of $\mathrm{Rh}$ immunoglobulin is given after the mother's first delivery, the development of kernicterus 
in subsequent infants can be prevented.

Perinatal cause: The two biggest risk factors for $\mathrm{CP}$ are prematurity and low birth weight. $A$ baby who is born prematurely and who weight less than $1500 \mathrm{~g}$ has a 25 to 31 times greater risk of developing CP than a typically sized newborn who weighs $3500 \mathrm{~g}$. A gestational age less than 37 weeks and small size for gestational age are compounding risk factors for neurologic deficit.

Postnatal cause: An infants or toddler may acquire brain damage secondary to cerebral hemorrhage, trauma, infection or anoxia. These conditions can be related to motor vehicle accidents, child abuse in the form of shaken baby syndrome, near drowning and inflammatory disorder of the brain like meningitis and encephalitis, which account for 60 percent of cases of acquired CP [11].

\section{CLASSIFICATION OF CEREBRAL PALSY}

The topographic classification of CP is monoplegia, hemiplegia, diplegia and quadriplegia. Monoplegia and Triplegia are relatively uncommon. There is a substantial overlap of the affected areas. In most studies, diplegia is the commonest form observed in 30\%-40\%, hemiplegia in $20 \%-30 \%$ and quadriplegia accounting for $10 \%-15 \%$ cases. It was found that spastic quadriplegia occur in $61 \%$ followed by diplegia in $22 \%$ on analysis of 100 cases with CP from india [1].

Quadriplegic CP: This is the most severe form involving all four limbs and the trunk, upper limbs are more severely involved than the lower limbs associated with acute hypoxic intrapartum asphyxia. However, this is not the only cause of spastic quadriplegia [12].

Neuroimaging reveals extensive cystic degeneration of the brain-polycystic encephalomalacia and polyencephaly. Voluntary movement are few: vasomotor changes of the extremities are common, most children have pseudobulbar signs with difficulty in swallowing and recurrent aspiration of food material. Half of the patients have optic atrophy and seizures intellectual impairment is severe in all cases [7].

Hemiplegic CP: Spastic hemiperesis is a unilateral paresis with upper limbs more severely affected than the lower limbs. It is seen in $56 \%$ of term infants and $17 \%$ of preterm infants. Pathogenesis is multifactorial, voluntary movement are impaired with hand function being most affected. Pincer grasp of the thumb, extension of the wrist and supination of the forearm are affected. In the loer limb, dorsiflexion and aversion of the foot are most impaired. There is increased flexor tone with hemiperetic posture, flexion at elbow and wrist, knees and equines position of the foot. Palmar grasp may persist for many years frequently, 2 point discrimination and position sense is also defective, seizures occur in more than $50 \%$ visual field defects, homonymous hemianopsia, cranial nerve abnormalities most commonly facial nerve palsies are seen [13].

Diplegic CP: Spastic diplegia is associated with prematurity and low birth weight. Nearly all preterm infants with spastic diplegia exhibit cystic periventricular leukomalacia on neuro imaging. Periventricular leukomalacia (PVL) is the most common ischeamic brain injury in premature infants. The ischeamia occurs in the border zone at the end of arterial $m$ vascular distributions. The ischeamia of PVL occur in white matter adjacent to the lateral ventricles. The diagnostic hallmarks of PVL are periventricular echo densities or cysts detected by cranial ultrasonography. Diagnosing PVL is important because a significant percentage of surviving premature infants with PVL develop cerebral palsy, intellectual impairment or visual distributions due to site of injury affecting the descending corticospinal tracts and visual radiations. Premature infants have impaired cerebrovascular auto regulation and are susceptible to intracranial hemorrhage $(\mathrm{ICH})$ as $\mathrm{ICH}$ detected on ultrasonography, maternal chorioamnionitis or vasculitis with production of cytokines, leading to inflammatory damage to the periventricular area in the developing brain is another factor in the pathophysiology of PVL. An estimated $60 \%-100 \%$ of patients with cystic periventricular leukomalacia go on to develop CP [14].

In this condition, lower limbs are more severely affected than upper limbs, mild cases 
may present with toe walking due to impaired dorsiflexion of feet with increased tone of the ankles. In severe cases, there is flexion of the hips, knees and to a lesser extent elbows. When the child is vertically, rigidity of lower limbs is most evidence and adductor spasm of the lower extremities causes scissoring of the legs. Siezures are common, fixation difficulties, nystagmus, strabismus and blindness have been associated with PVL.

\section{Types of neuromuscular deficits in CP}

1) spastic

2) dyskinetic (including choreoathetoid and dystonis)

3) Ataxic

4) Hypotonic

5) mixed

Spastic CP is the most common and accounts for $70 \%-75 \%$ of all cases. Dyskinetic $10 \%-15 \%$ and ataxix is less than $5 \%$ of cases spatic type exhibit pyramidal involvement with upper motor neuron signs, weaknesis, hypertonia, hypereflexia, clonus and positive Babinski. Dyskinesia is characterized by extra pyramidal involvements in which rigidity, chorea, choreaoathetosis, athetoid and dystonic movements are seen. This type of CP is also associated with birth asphyxia. [12] The severity of dystonic postures may vary with body positions, emotional state and sleep clonus and Babinski sign are absent. Primitive reflexes are more prominent and persistent for a longer time in dyskinetic CP. These movement patterns are eliminated in sleep, with a decrease in tone of the affecte limbs. There are also abnormalities of posture control and coordination. These children who are hypotonic to start with may develop into this type by 1 to 3 years of age. In majority of this group, there is no cognitive impairment. Dysathria, oromotor problems with drooling and swallowing difficulties are seen, $30 \%$ of children with CP have a mixed pattern of involvement while contractures are common in spastic group they are uncommon in the extra pyramidal group. Hypotonic $\mathrm{CP}$ is characterized by generalized muscular hypotonia that persists beyond 2 to 3 years of age that does not result from a primary disorder of muscle or peripheral nerve. The deep tendon reflexes are normal or hyperactive and the electrical reactions of the muscle and nerve are normal. More than half the children develop frank cerebellar deficits with incoordinatinated ataxia and impaired rapid successation movements.

\section{PATHOPHYSIOLOGY}

Table 2: Correlation of pathophysiological cause to neurological deficit noted in CP.

\begin{tabular}{|c|c|c|}
\hline S. No. & Cause & Deficit \\
\hline 1 & Intraventricular hemorrhage & Spastic diplegia \\
\hline 2 & Hypoxic ischeamic injury & Spastic quadriplegia \\
\hline 3 & Selective neuronal necrosis of cerebellum & Ataxia \\
\hline 4 & $\begin{array}{c}\text { Status marmoratus (hyper myelination in } \\
\text { basal ganglia) }\end{array}$ & Athetosis \\
\hline
\end{tabular}

Spastic diplegia, quadriplegia can caused by varying degrees of intraventricular hemorrhage or hypoxic - ischeamic injury. Depending on which fibres of corticospinal tract are involved and whether the damage is bilateral or unilateral the resultant neurologic deficit manifests as quadriplegia, diplegia or hemiplegia. Spastic quadriplegia is most often associated with grade iii intraventricular hemorrhage in immature infants, what used to be classified as grade iv hemorrhage is now called periventricular hemmorhagic infarction (PHI). Preterm infants with low birth weights who have $\mathrm{PHI}$ are substantially higher risk for neurological problems. Premature infants born at 32 weeks of gestation are especially vulnerable to white matter damage around ventricles from hypoxia and ischeamia. Periventricular leukomalacia (PVL) is most common cause of spastic diplegia because the fibres of the corticospinal tracts that go to the lower extremities are most exposed. Spastic hemiplegia, the most common types of $\mathrm{CP}$ can result from unilateral brain damage secondary to an intraventricular hemorrhage or other hypoxic events. Selective neuronal necrosis can result from hypoxic ischeamic injury in the mature neonate [15].

Athetosis involvesdamage to the basal ganglia and has been associated with erythroblastosis fetalis, anoxia and respiratory distress. Erythroblastosis, a destruction of Red blood cells, occurs in the newborn when an Rh incompatibility of maternal-fetal blood groups 
Table 3: Gross motor classification system.

\begin{tabular}{|c|l|}
\hline Level & \multicolumn{1}{|c|}{ Abilities and limitations } \\
\hline I. & Walks without limitations at home, school, outdoors, and in the community \\
\hline II. & Walks without assistive devices but has limitations walking outdoors and in the community \\
\hline III. & $\begin{array}{l}\text { Walks using a hand-held mobility device (cane, crutches, walker). Limitations walking outdoors and in the community - child } \\
\text { transported in a wheelchair or uses powered mobility }\end{array}$ \\
\hline IV. & $\begin{array}{l}\text { Child uses wheeled mobility in most settings; requires adaptive seating and physical assistance for transfers. Self-mobility can } \\
\text { be achieved using powered mobility }\end{array}$ \\
\hline V. & $\begin{array}{l}\text { Self-mobility is severely limited even with the use of assistive technology. Child may achieve self-mobility using powered } \\
\text { mobility with extensive adaptations for seating and control access }\end{array}$ \\
\hline
\end{tabular}

Table 4: The manual ability classification system (MACS).

\begin{tabular}{|c|l|}
\hline Level & \multicolumn{1}{|c|}{ Abilities and limitations } \\
\hline I. & $\begin{array}{l}\text { Handles objects easily and successfully. At most, instead of handling objects with both hands, may have limitations in } \\
\text { performing manual tasks requiring speed and accuracy. However, any limitations in manual abilities do not restrict } \\
\text { independence in daily activities. }\end{array}$ \\
\hline II. & $\begin{array}{l}\text { Handles most objects but with somewhat reduced quality and/or speed of achievement. Certain activities may be avoided } \\
\text { or be achieved with some difficulty; alternative ways of performance might be used but manual abilities do not usually } \\
\text { restrict independence in daily activities. }\end{array}$ \\
\hline III. & $\begin{array}{l}\text { Handles objects with difficulty; needs help to prepare and/or modify activities. The performance is slow and achieved } \\
\text { with limited success regarding quality and quantity. Activities are performed independently if they have been set up or } \\
\text { adapted. }\end{array}$ \\
\hline IV. & $\begin{array}{l}\text { Handles a limited selection of easily managed objects in adapted situations. Performs parts of activities with effort and } \\
\text { with limited success. Requires continuous support and assistance and/or adapted equipment for even partial } \\
\text { achievement of the activity. }\end{array}$ \\
\hline V. & \begin{tabular}{l} 
Does not handle objects and has severely limited ability to perform even simple actions. Requires total assistance. \\
\hline
\end{tabular}
\end{tabular}

exists. Ataxia is related to damage to the cerebellum [15].

Impairment: The primary motor impairments of cerebral palsy are the result of an upper motor neurone lesion leading to weakness, spasticity, contracture, in coordination, altered sensation and balance, and associated reactions. Although cerebral palsy itself is non-progressive, progressive motor impairment is a feature which often becomes more noticeable during periods of growth and development [16]. As a child with cerebral palsy ages, they tend to develop increasingly abnormal movement along with accompanying increases in spasticity and contracture. These changes lead in turn to a general poverty of movement as a result of muscular atrophy, disuse and stretch weakness. Long term musculoskeletal changes can be seen into adulthood with restricted muscle ledislocation. In addition, cartilage damage, which occurs through altered compression and immobility, results in osteoporotic changes and painful arthritis [3]. Therefore, the effect that these impairments have on developing skeletal muscles, and subsequently the motor performance of children with cerebral palsy, is of most concern to physiotherapists.

Activity limitation: The presence of impairments in children with cerebral palsy is likely to have a carryover effect into their everyday physical activities such as walking, climbing on play equipment and moving around at school and the community. Correspondingly, these children are more likely to engage in sedentary behaviours and therefore be less active than their typically developing peers [17]. The Gross Motor Classification System (GMFCS) was developed to classify the amount of activity limitation seen in children with cerebral palsy and describes their activity in relation to walking. This system comprises 5 levels to best represent the child's current level of physical activity and mobility in their home, school and community settings [3]. The GMFCS has been widely adopted for use in both research and clinical settings.

The Manual Ability Classification System (MACS) classifies the amount of upper limb activity in children with cerebral palsy [18] (Table 4). The five levels in the MACS are based on the child's self-initiated ability to manipulate objects and their need for assistance or adaptation to perform manual activities in everyday life.

Participation restriction: The World Health Organisation (WHO) defines participation restriction as problems an individual may experience when attempting to be involved in a life situation. For children with cerebral palsy, this may mean difficulties when walking to a 
classroom, interacting with their peers during lunch and play breaks or participating fully in school physical education programs. Participation is very much an individual choice of activity and can be categorised by aspects of performance and capacity. For example, what a child can do (their performance), ie, such as walking measured using a 10-m Walk Test or a 6 Minute Walk Test, versus what they actually do (their capacity) ie the amount of physical activity carried out each day. The difference between performance and capacity is most likely to be dependent on personal and environmental factors such as choice or availability of resources. Subsequently, participation restriction is difficult to measure and is most commonly assessed by determining either the child's, or parents', perception of their quality of life. For a child, their perception of quality of life may be determined by their ability to interact and be involved in activities with their typically-developing peers. For parents of a child with cerebral palsy, the stress and everyday demands of caring for a child with a disability may determine their perceptions. It is recognized that there are many potential causes of poor quality of life factors which can impact on the child, their parents or caregivers, and subsequent family functioning. It is also likely that these factors are greater as the severity of cerebral palsy increases [19].

\section{DIAGNOSIS}

Children with $\mathrm{CP}$ are more likely to have associated conditions including mental retardation (52\%), ophthalmologic defects (28\%), hearing impairment (12\%) and speech and language disorders (38\%) and additional oral-motor deficits. Children with $\mathrm{CP}$ are also greater risk for epilepsy (mean $43 \%$; range $34 \%$ to $94 \%$ ) However, there is no evidence that EEG helpful in determining the etiology of CP.

Recommendation for the evaluation of children with CP. Although there is insufficient evidence to recommend the optimal sequence of tests to determine the etiology of $\mathrm{CP}$, taking into account diagnostic yield and potential treatability, we propose the following consensusbased evaluation as outlined in the alg rithm. All children should undergo a detailed history and physical examination. It is important to determine that the childs condition is due toa static and not a progressive or degenerative neurologic disorder. It is als important to classify the type of $\mathrm{CP}$ as this has diagnostic implications as well as implications regarding associated problems. Because children with $\mathrm{CP}$ commonly have associated mental retardation, ophthalmologic abnormalities, hearing impairments, speech and language disorders and disorders of oral-motor functions, screening for these conditions should be part of the initial assessment. An EEG is recommended when there are features suggestive of epilepsy syndrome. In order to establish ban etiology and prognosis in children with CP neuroimaging is recommended with Magnetic Resonance Imaging (MRI) preferred to Computed Tomography (CT). However if neuroimaging performed in the perinatal period provided an etiology of the child $C P$, it may obviate the need for later study. Metabolic and genetic studies should not be routinely obtained in the evaluation of the child with CP. If the clinical history or findings on neuroimaging do not determine a specific structural abnormality or if there are additional and atypical features in the history or clinical examination, met bolic and genetic testing should be considered.Because the incidence of cerebral infarction is high in children with hemiplegic $C P$, diagnostic testing for a coagulation disorder should be considered [20].

\section{Physical therapy examination/assessment:}

In an assessment of a child, whose physiotherapy and rehabilitation needs were determined and then was sent to a physiotherapist, answers of questions such as "Why is physiotherapy required?", "What are the active neurophysiologic and biomechanical mechanisms?", "How do the accompanying problems effect the situation?" should be searched. The clinical type, severity of the disease, chronologic age, age of initiating physiotherapy, existence and severity of abnormal reflexes, cognitive problems appearing together, hearing disorders, visual impairment, sensory-perception problems, general state of health and the sociocultural and economic status of the family should be considered while deciding on suitable physiotherapy 
methods [21]. The actual question that needs to be answered within scope of the information obtained, as a result of the assessment, is what is important in the child's life. What needs to be provided is not only motor development abilities such as sitting, crawling, walking, muscle tonus regulation, balance and coordination training. The acquisitions shall be ensured to be able to be used in daily life [19]. While clinical observation is one of the most important parts of the assessment, it completes standardized tests and contributes information which carries at least the same significance.

By assessing the child, according to the parameters listed below, the physiotherapist shall present a general table of the child. The child must be calm and trust the physiotherapist during the observations conducted in terms of motor, sensory, cognitive, emotional and social/family. The mother, father or the guardian undertaking the care of the child shall be with the physiotherapist during the observation. The child must not be hungry, nor should be observed right after eating. The room where the observation will be done should be quiet, at an agreeable temperature and not contain unnecessary toys and equipment; if possible it should be a room covered with material that is appropriate for the child to move on the ground, with walls painted in warm colours and should not be too small.

Firstly, what the child can do on his/her own should be observed while examining the functional movements, fine and gross motor skills during the observation [22]. Within the scope of the assessment to be performed in terms of motor, besides the changes in the muscle tonus, co-contraction capacities of the muscles, involuntary extremity and body movements, stabilisation of the extremities, correction, balance and protective reactions, sitting balance, upper extremity and hand functions and sensory-perception problems; orthosis, need of mobilisation tools and other aid tools, cooperation of the family and their knowledge on the disease also needs to be assessed. The assessment of the motor function should be based on the normal process of a normal motor function development but it should also be sensitive towards special problems. For motor development reflex development, proper posture, sufficient extremity movements, appropriate muscle tonus, sensory development and cognitive functions within an integral neurologic and musculoskeletal system is required. Full completion of the motor development is required for the functional independence and social and emotional development of the child. Therefore it is required to know the normal development of a child.

By knowing the normal development, the developmental problems that may occur in the child due to any reason can be better understood [23]. The methods used for assessing spasticity take place within a wide range that extends from clinical scales to more complex systems based on Electromyographic Analysis (EMG). Collecting comprehensive history and observations are very important in assessing the effect of spasticity on functions.

The muscle groups, in which the spasticity exists, and their interaction with postural reactions' effect on functions should be researched. Although assessing functional activities and daily-life activities does not directly determine the severity of spasticity, it could present an idea on the reflection of the changes of the spasticity on the functional condition. One method of assessing spasticity in the clinic is to determine the amount of resistance that the spastic muscle presents during a passive movement of the relevant extremity. Ashworth has, accordingly, defined a 5-point scale. This scale evaluates the resistance that occurs during the passive movements of the extremities with points between 0-4. Although the Modified Ashworth Scale (MAS) is a subjective method in our day, it is widely used as an easily applied method that does not require any tool in assessing spasticity. The Tardieu Scale is another scale that assesses spasticity with passive movements, as does the AS and MAS. This scale presents spasticity's nature that depends on speed. Passive straining is performed at the speed of the extremity segments falling with gravity and slower and faster than this speed. The Modified Tardieu Scale (MTS) has added the assessment positions and spasticity angles of the extremities to the original scale. The MAS, Pendulum test and MTS for 
measuring the spasticity of children with $\mathrm{CP}$ was compared and MTS was determined to be most appropriate measurement method [18]. Spastic muscles limit articular movements in antagonist directions. Therefore, in addition to assessing the movement of the articular with a goniometre can also be used as an objective method although it presents conflicting results in terms of reliability.

Assessments, which are not widely used in the clinic and are used more in assessments researches, are methods such as the dynamic flexometre,pendulum test,electrophysiologically assessing the $\mathrm{H}$ reflex and $\mathrm{M}$ response and the biomechanical analysis of response of the spastic muscle to angular and speed differences, etc. [23]. The Barry Albright dystonia scale is a highly reliable rating scale developed in order to asses the dystonia in patients with $\mathrm{CP}$ and traumatic brain injuries. The scoring is "none"; 0, "slight"; 1, "mild"; 2, "moderate"; 3, and "severe"; 4 . Each region has specific descriptors for a scoring. Generally if dystonia is present less than $10 \%$ of the time it is "slight", if it does not interfere with function or care it is "mild", if it makes functional movements harder it is "moderate", and if it prevents function it is "severe" [24]. The changes in the muscle tonus must include the contraction capacity of the muscles, involuntary extremity and trunk movements, the stability of the trunk and extremities, correction and balance reactions, sitting balance, upper extremity and hand functions, sensory-perception problems, speech and tongue functions and dietary status. Orthosis, mobilisation and other adaption devices, the general health condition of the child and the family's socio-cultural and economical conditions should also be assessed. Various tests and batteries should be used in order to assess different parameters such as the motor and reflex development level, muscle strength, normal articular movement, functional skills, and independence level and self-care activities [23]. The most widely-used test battery that measures the functional motor level in order to determine the motor development level of children with CP is the Gross Motor Function Measurement (GMFM). With GMFM, physiotherapists can define the motor function level of the child; obtain aid in specifying the targets of the treatment, follow-up the post treatment development and present objective information regarding the child to relevant colleagues, other inter-discipliner professionals and families. It was developed in 1989 by Russell et al. by considering the motor function level of a 5-year old child with normal motor development. The GMFM measures how much of the action is achieved rather than measure the quality of the motor performance. The purpose is to determine the capacity and change. It is comprised of sections of supine-facedown positions and turning, sitting, crawling and standing on knees, standing on feet, walking and running and jumping [19]. The Gross Motor Function Classification System (GMFCS) is a classification system developed for children with CP. The GMFCS has been developed by Palasino based on the actions the child can perform from sitting to walking. It is a practical system that can be used in clinics for the rehabilitation team to classify a child with $\mathrm{CP}$, observe the efficiency of the applications and follow-up on the patient in inter-intra discipliner applications. Initially, children with $\mathrm{CP}$ aged below 12 were divided into five levels by considering their independency in gross motor functions such as sitting, walking, mobilization and transfer activities and the tools-equipment, tools that assist in walking that they use.

As motor functions of children differ according to age, functions have been defined as under 2-years old, between 2-4 years old, between 4-6 years old and between 6-12 years old for each level. This system was extended in order to include the age ranges of between 12-15 and 15-18 years old in 2007. The Manual Ability Classification System (MACS) is a system developed in 2003 in order to classify the ability to hold objects with hands during daily activities of children with $\mathrm{CP}$ between the ages of 4-18. MACS aims to specify which level represents best the child's normal activities at school, home and in society. The MACS classification should be specified based on the real performance of the child in daily life. This should not be done by performing a special assessment, but by asking a person who knows the child and how he/she generally achieves these. While 
specifying the MACS level, it should be kept in mind that the child's ability of holding objects is related to age. The MACS assesses the participation of both hands in an activity and cannot separately asses the hand functions. The aim of the MACS is to provide a systematic method regarding classifying how children with $\mathrm{CP}$ use their hands while holding objects during daily activities. MACS, focuses on manual abilities that are initiated by the person itself and especially on holding the objects surrounding them. The MACS has defined 5 levels. The differences between each level also help to specify the level which has the closest similarity to the child's manual abilities [25].

The Functional Independence Measure for Children = WeeFIM has been developed by using the Functional Independence Measure (FIM) developed for adults by "Uniform Data System" in 2003. It is a useful, short, comprehensive measurement method that determines the development, educational and social functional limitations of children that have CP and other development disorders. WeefIM contains a total of 18 articles in 6 fields; selfcare, sphincter control, transfers, locomotion, communication, and social and cognitive. Whether or not the child is aided, performs on time or if they required an aiding device while performing the function in each article of these fields is scored from 1 to 7.1 point is given if they perform the mission with aid, 2 for independently performing, and 7 if they perform on the right time and safely. Accordingly, the child can score 18 the least (fully dependant) and 126 the most (fully independent) [26].

The Pediatric Evaluation Disability Inventory (PEDI), is a comprehensive clinical assessment tool that assesses the functional ability and performance of disabled children. It has been developed especially to assess the function of small children and is a distinguishing measurement method that can be used for children below 7, 5 years old and also older children. PEDI, is comprised of three main sub-sections; functional abilities, help of caretakers and modifications. Each of these sections assesses self-care, mobility and social function areas. The functional abilities part comprises of 197 articles and measures the functional abilities of the child. In this section the "self-care" sub-section comprises of 73, the "mobility" sub-section comprises of 59 and the "social functions" sub-section comprises of 65 articles. The section regarding help of the caretakers comprises of 20 articles and measures the disability condition of the child according to the amount of aid required in order to perform the functional activity. The modifications section also comprises of 20 articles and shows the environmental modifications and tools that the child uses during his/her daily life. Each sub-section of PEDI can be used independently [19].

Health-related quality of life (HRQL) is defined by the World Health Organisation Quality of Life Group as "an individual's perception of their position of life in the context of the culture and value systems in which they live and in relation to their goals, expectations, standards, and concerns". The International Classification System (ICF) notes that the HQRL should be taken into consideration in the assessments. Health-related quality of life should be considered in children with $\mathrm{CP}$ by terms of the child and the family. HRQL assessment in children with CP, should mostly contain physical symptoms, activity limitations, emotional stress, communication problems between the child-family, limitation of school life and difficulties experienced in the treatment of the disease. Due to the CP chart of children with $C P$, pain, general health, physical functions, actions with family are HRQL parameters. In comparison to normal children, as a result of $C P$, decrease of independency at a functional level, difficulty in daily life activities, and cognitive and sensory problems that may accompany negatively affect the HRQL in children with CP. Cognitive levels and speech functions are significant while determining the HRQL of children under 18 . Therefore, surveys that measure the family's influence from the child with CP are widely used while determining the child's HRQL. It is required to develop quality of life questionnaires that comprise of questions that children that do not have cognitive problems can answer on their own. When literature is examined, we see that while assessing the HRQL of children with $C P$, Paediatric 
Outcomes Data Collection Instrument (PODCI) and Child Health Questionnaire (CHQ) are used the most.

The Child Health Questionnaire-Parent Form (CHQ-PF50); is an assessment method developed to assess the HQRL of children aged between 5 and 18 [17]. Besides motor function problems being the main problem, when sensory, cognitive and mental problems accompany, the families' and caretakers', who have to take care of the children with $\mathrm{CP}$, whose daily life functions and independencies are affected, all day for many years, are also affected in physical and psycho-social terms. In terms of physical and psychosocial health, the child with CP's general health, the parent's own psychological structure, social support and distribution of roles within the family are influential in the family's HRQL. General health profiles and tests that measure HRQL are mostly used for measuring the influence on families with children with CP [18].

\section{PHYSIOTHERAPY MANAGEMENT OF CEREBRAL PALSY}

The aim of rehabilitation in children with $\mathrm{CP}$ are; to minimize the effect of physical impairments, to gain independence in the community and to improve the quality of life of the handicapped children and their families who have major roles to play in the process [21]. Rehabilitation in children with CP can differ due to clinical type and severity of table, additional disabilities, and physiological age of children socioeconomic factors. In addition visual, auditory, cognitive disorders, seizures, learning disabilities and emotional problems may influence intervention outcomes [27].

The physiotherapist focuses on gross motor skills and functional mobility in the management for the motor deficits in CP. Positioning, sitting, transition from sitting to standing, walking with or without assistive devices and orthoses, wheelchair use and transfers, are areas that the physiotherapist works on. In most settings the physiotherapist performs therapy, plans the home program, provides the interphase with the school and recommends equipment [28]. Physiotherapists emphasize the need for the practice to be evidence-based whenever possible. Recently, reviews have addressed the Int J Physiother Res 2018;6(5):2864-81. I ISSN 2321-1822 effectiveness of physiotherapy interventions for children with CP focusing on neuro-developmental therapy (NDT), strength training, conductive education, various physiotherapy interventions and orthotic management [24].

Management of motor disability: Early intervention programs address developmental deviations that result from associated central nervous system deficits or are imposed by impaired motor function.9 Public Law 99-457 (Education of the Handicapped Act of 1986) is a federal mandate to provide such programs for high-risk Infants.

Therapies for neuromuscular disorders in infants and young children incorporate handling, positioning, and play to challenge postural and movement responses that are prerequisites for gross and fine motor milestones. Feeding difficulties require the facilitation of oral motor coordination to improve swallowing. Instructing the family in modified ways of physical handling extends therapeutic efforts to everyday activities at home [29]. Therapy becomes more structured as children learn to follow instructions and are able to co operate. During the preschool years, the functional goal is to achieve the most effective mode of locomotion and selfcare skills, with assistive devices if necessary.

Compensatory functional training includes a range of rehabilitation possibilities that can be illustrated by several examples. Environmental modifications to ensure access, suitable clothing, and learning to use simple adapted implements for feeding, writing and other daily tasks are practical approaches to independence that occupational therapy offers [16]. Although assistive devices such as crutches, walkerettes, and wheelchairs have been used for a long time, rehabilitation engineering has revolutionized the technology for disabled persons. The light weight and versatility of new wheelchair designs allow greater mobility, including participation in adapted sports. An important development for patients with cerebral palsy is the construction of wheelchair seats that incorporate biomechanical features to alleviate abnormal tone and posture [19]. When upper extremity impairment necessitates, motorized wheelchair models are available from pediatric to adult sizes. Children with adequate cognitive, visual, and spatial 
discrimination abilities can learn to operate a motorized device under adult supervision as early as 3 years of age. Electronic and computer technologies have created a range of possibilities to help patients overcome physical restrictions in activities of daily living, education, work, and leisure.9"I' Economic constraints are the primary obstacle that limits the availability of such devices.

Preventing musculoskeletal complications, which can further compromise function, constitutes an additional aim of treatment [20]. Abnormal tone, movements, and posture create a risk of soft tissue contractures and bone deformities. The propensity for these complications is greatest during the rapid phases of growth in childhood, but an insidious progression may continue after skeletal maturity [21]. Anticipatory treatment is the best preventive measure. Options include range-of-motion exercises, splinting, braces, and surgical procedures as warranted by the clinical findings and course.

Therapy is most intensive from infancy to early school years because most children reach their expected functional capacity in ambulation and daily activity skills by school age [22]. As formal therapy is gradually tapered, consistent practice at home and school and in other functional situations must continue to increase motor proficiency, endurance, and the self-initiated acquisition of new skills. Adapted physical education and sports, aside from their benefits on motor abilities, provide enjoyment, companionship, and a sense of accomplishment, which are important for the development of confidence and self-image in all children and adolescents [23]. A periodic reinstitution of formal therapy may be necessary for defined reasons, such as to regain preoperative function after immobilization for orthopedic procedures or to learn new skills that have become appropriate and feasible for the child's developmental level and physical abilities.

Lower extremity orthoses or splints are used to improve function-for example, toe walking-or to prevent deformity in a child who does not ambulate [24]. Extensive bracing does not enable a child with severe motor disability to attain useful walking. Despite these considerations, the treatment team might elect to use orthoses for passive standing or exercise ambulation in a bright, well-motivated youngster. The family, however, must understand clearly the limited purpose of these attempts.

\section{Speech and language impairments manage-} ment: Speech and language development need close monitoring because effective communication is one of the most valuable functional assets [25]. All children with cerebral palsy should have an audiologic examination and a timely correction of hearing impairments that may have detrimental effects on language acquisition. Sensorineural hearing loss is the characteristic auditory deficit and is particularly common in patients with kernicteric athetosis. Central language disorders are more likely related to cognitive dysfunction because young children show good recovery from aphasia after suffering damage to the dominant hemisphere. A subtle impairment of language competence may persist despite fluent speech, however, and also has been reported with congenital right hemiparesis [26]. Speech defects occur in athetosis and bilateral spastic paresis when pseudobulbar palsy affects the coordination of muscles that participate in phonation and articulation [27]. The resultant speech impairments range from mild articulation disorder to severe dysarthria with a total lack of functional verbal communication.

Therapy for speech and language deficits should commence as soon as the deficits are detected. An important advance in the treatment ofsevere speech defects is nonverbal communication [28]. The simplest initial method is the simultaneous presentation of several objects or an improvised communication board of pictures from which a child can select the appropriate response by eye gaze, pointing, or any other consistent signal. When education and social interaction demand an expanded repertoire of communication skills, manually or electronically operated voice and printed word-symbol communicators offer various choices [29]. The potential for nonverbal communication should be explored when speech is delayed to identify children who have sufficient cognitive language ability to master this mode of interaction.

Intervention to improve to sensory integration: The theory of sensory integration was 
for passive standing or exercise ambulation in a bright, well-motivated youngster. The family, however, must understand clearly the limited purpose of these attempts.

Speech and language impairments management: Speech and language development need close monitoring because effective communication is one of the most valuable functional assets [25]. All children with cerebral palsy should have an audiologic examination and a timely correction of hearing impairments that may have detrimental effects on language acquisition. Sensorineural hearing loss is the characteristic auditory deficit and is particularly common in patients with kernicteric athetosis. Central language disorders are more likely related to cognitive dysfunction because young children show good recovery from aphasia after suffering damage to the dominant hemisphere. A subtle impairment of language competence may persist despite fluent speech, however, and also has been reported with congenital right hemiparesis [26]. Speech defects occur in athetosis and bilateral spastic paresis when pseudobulbar palsy affects the coordination of muscles that participate in phonation and articulation [27]. The resultant speech impairments range from mild articulation disorder to severe dysarthria with a total lack of functional verbal communication.

Therapy for speech and language deficits should commence as soon as the deficits are detected. An important advance in the treatment ofsevere speech defects is nonverbal communication [28]. The simplest initial method is the simultaneous presentation of several objects or an improvised communication board of pictures from which a child can select the appropriate response by eye gaze, pointing, or any other consistent signal. When education and social interaction demand an expanded repertoire of communication skills, manually or electronically operated voice and printed word-symbol communicators offer various choices [29]. The potential for nonverbal communication should be explored when speech is delayed to identify children who have sufficient cognitive language ability to master this mode of interaction.

Intervention to improve to sensory integration: The theory of sensory integration was originally developed by A tean Ayres in the 1970s [30]. The principles of SI theory are used by therapist in developing treatment approaches for children with sensory processing difficulties including CP As concived by Ayres the SI model was develop to treat learning disabilities. SI theory is based on the hypothesis that in order to develop and execute a normal adaptive behavioral response, the child must be able to optimally receive modulate integrate and process the sensory information [25] many children with learning disabilities cerebral palsy and other neuro developmental disabilities have associated sensory difficulties. The SI approach attempts to facilitate the normal development and improve the childs ability to process and integrate sensory information (visual, perceptual, proprioceptive, auditory etc). It is proposed that this will allow improved functional apabilities in daily life activities. As originally described by Ayres the objective of SI Approach is not to teach specific skills but "to enhance the brain capacities to perceive, remember and motor plan. A therapeutic environment is created in which the child gains rich sensory motor experience. The therapist engages the child in challenging play activities in such a way that the child is able to overcome the challenge and adapts to subsequently face more challenging stimulus. The therapist takes cues from the childs behavior and provide appropriate sensory rich play environment.

The therapist works closely with other members of the intra discipilinary team, so that an appropriate challenging sensory motor experience can be provided for the child in daily life settings and the functional capabilities can be monitored. Three classic patterns of SI disorders have been proposed namely sensory modulation, sensory discrimination disorders and sensory based motor disorders. Such grouping is intended to guide formulation of specific intervention for homogenous disorders overall some studies find $\mathrm{SI}$ as a useful treatment approach in children with $\mathrm{CP}$ while others do not find any functional benefit.

Management of feeding difficulties: Feeding difficulties and salivation are additional signs of pseudobulbar palsy. Skillful feeding and carefully selected food textures facilitate oral 
motor function [29].

In almost all patients, dysphagia and drooling are reduced with time. In severe cases, inadequate nutritional intake or recurrent aspiration necessitates temporary or permanent feeding gastrostomy. For the socially embarrassing handicap of drooling, there are behavioral, pharmacologic, and surgical treatment methods.

Management of spasticity: Treatment for the child with spasticity focuses on mobility in all possible postures and transitions between these postures. The tendency to develop contractures needs to be counteracted by range of motion, positioning, and development of active movement. Areas that are prone to tightness may include shoulder adductors and elbow, wrist, and finger flexors in children with quadriplegic involvement, whereas hip flexors and adductors, knee flexors, and ankle plantar flexors are more likely to be involved in children with diplegic involvement. Children with quadriplegia can show lower extremity tightness as well. These same joints may be involved unilaterally in hemiplegia. Useful techniques to inhibit spasticity include weight bearing; weight shifting; slow, rhythmic rocking; and rhythmic rotation of the trunk and body segments. Active trunk rotation, dissociation of body segments, and isolated joint movements should be included in the treatment activities and home program. Appropriate handling can increase the likelihood that the child will receive more accurate sensory feedback for motor learning [30].

Body weight support treadmill training: Stepping movements (or reflex stepping reactions) are normally present in newborns and infants before the infants start to bear weight, stand and walk [26]. In treadmill training the child is supported in an upright posture limiting weight bearing. The child then attempts to walk on the slowly moving treadmill, eliciting the stepping movements. Treadmill training thus allows development of stepping movements needed for ambulation. Studies shown improvement in lower extremity movements and gait patterns in children with cerebral palsy. Neuro developmental training (NDT): This therapeutic approach was developed by Berta and Karl Bobath in the 1940s, based on their personal observations working with children with cerebral palsy. 6"1z It is one of the more popular therapeutic interventions for the treatment of children with CP. There are thousands of trained therapists all over the world using this approach, who are trained at various NDT training centers and NDT courses? The basis of NDT approach as conceptualized by Bobaths is that the motor abnormalities in children with CP are due to failure of normal development of postural control and reflexes because of the underlying dysfunction of the central nervous system. The aim of the NDT approach is to facilitate normal motor development and function and to prevent development of secondary impairments due to muscle contractures and joint and limb deformities. Originally, the Bobath approach used various techniques to inhibit and control abnormal tone, reflexes and movement patterns J2. This was postulated to facilitate normal postural and righting reflexes, and movement patterns. The normal developmental sequence of child development is used as the underlying guiding principle. It was postulated that such normal therapeutic experience in automatic movements and reflexes will translate into the child developing normal tone and volitional movements with improved functional capabilities. With further experience, the Bobaths noted that there was a lack of such carryover effects, and modified their approach so as to allow the child to take over more control of balance and movement, treat children in natural play environments, and not necessarily to follow rigid developmental sequence. There is a wide variation in the expertise and training of therapists who use NDT approach with various modifications. There are also significant differences in the NDT application in different countries. Typically, most sessions are of I hour duration each, and given at least 2 times per week- $\sim$ z Intensive NDT has been practised by some with 1 hour per day for 5 days per week and reported to be more effective. The parents and caregivers are also trained to continue the therapy at home during daily activities and play. Various therapeutic aides such as orthotics and balls are used as necessary. NDT approach takes long-term view recognizing the need for continued intervention to maintain functional capabilities and prevent deformities and contractures. Although the effectiveness of NDT 
in $\mathrm{CP}$ has been questioned by some published reports, there are some studies suggesting its effectiveness.

CIMT: Constraint-induced therapy is used to improve the use of affected upper extremity in a child with hemiplegic CP. The normally functioning or stronger upper extremity is immobilized for a variable duration in order to force the use of the affected or weaker upper extremity over time. The efficacy of this approach has not been established, and adverse effects of prolonged immobilization of the normally developing upper extremity are a significant concern.

\section{DISCUSSION}

The aim of this review was to examine the evidence available for physiotherapy intervention for children with cerebral palsy. Despite advances in medical intervention and rehabilitation, cerebral palsy is the most common activity-limiting condition in children and adolescents worldwide. Likewise, the motor impairments and activity limitations associated with cerebral palsy continue to be the primary focus for physiotherapy intervention. Determining the most effective form of physiotherapy intervention to address motor impairments and activity limitations in order to promote physical activity and enable community participation in children with cerebral palsy, is therefore an important issue for physiotherapists.

Since the studies in this review have been conducted over some time, an update is given of how the evidence for physiotherapy intervention for children with cerebral palsy has progressed, and future directions are proposed.

\section{EVIDENCE FOR PHYSIOTHERAPY INTERVEN- TION - Study 1}

\section{Where were we in 2017?}

Study 1 was a summary of systematic reviews which identified 8 systematic reviews examining the effects of 6 physiotherapy interventions for children with cerebral palsy. While the reviews were deemed to be of high quality, the low number of randomized controlled trials within each review prevented meta-analysis. Consequently there is little evidence for use by clinicians to direct their practice. In addition, the evidence identified was not convincing as there were contradictory results at both the impairment and activity levels and no evidence at the level of participation. For example, the evidence in one review that a physiotherapy intervention was beneficial was contradicted by evidence in another review that it had no effect. The strength of evidence was further undermined by the fact that most of the reviews contained studies of other design as well as randomized controlled trials.

Only one trial in one review reported severity of disability as determined by the Gross Motor Function Classification Scale (GMFCS) [7] therefore the similarity of the participants between studies could not be assessed. Furthermore, no conclusions could be made regarding the effect of intervention on the level of severity of cerebral palsy and the lack of significant findings could be attributed to the diverse range of abilities within these participants. Including the most appropriate level for the intervention, ie, choosing Level I and/or II GMFCS, for investigation of walking ability may be more appropriate. A further issue was identified with the choice of outcome measures used particularly at the activity level.

For example the Gross Motor Function Measure (GMFM) [17] was developed as a measure of motor performance but in many studies was frequently used to reflect amount of activity. The failure of many studies to find any carryover to activity may be more reflective of the outcome measures used rather than the intervention.

\section{EVIDENCE FOR PHYSICAL ACTIVITY - Study 2}

\section{Where are we now?}

In Study 2, the amount of moderate to vigorous physical activity performed by ambulant children with cerebral palsy was examined and compared to their age matched peers. These children had mild impairments which would theoretically enable them to engage in a normal amount of physical activity. The findings from this study showed that children with cerebral palsy were capable of performing moderate to vigorous physical activity. They were spending a similar amount of time each day in physical activity to their peers. However, the amount of daily physical activity for both groups was less than the 
recommended guidelines (Department of Health and Ageing 2005) of 60 minutes moderate to vigorous Activity. There are no other studies using accelerometers which compare physical activity with typically-developing peers. However, while this study was being carried out, two studies $[14,19]$ which examined moderate to vigorous physical activity in children with cerebral palsy using an accelerometer were published. The period of activity measured in both these studies was relatively short, ie, $15 \mathrm{~min}$ utes in study [14], and 22 minutes total in the study [19]. The activity measured was walking, circuit-type activities and free play. In line with Study 3 , the findings from these two studies showed that children with cerebral palsy were able to achieve moderate to vigorous amounts of physical activity.

\section{BARRIERS TO PHYSICAL ACTIVITY - Study 3}

\section{Where are we now?}

This study examined the barriers to physical activity in children with cerebral palsy from the parents' perspective. These children had mild impairments which would theoretically enable them to engage in a normal amount of physical activity. The findings from this study showed that children with cerebral palsy were regularly engaged in the type of physical activities which are fairly representative of typically developing children. However, parents perceived many barriers which were either preventing their child from being engaged in physical activity, or from performing physical activity more frequently. The cost of activities was perceived by parents as being the greatest barrier to more frequent participation in activities. Where do we need to go in the future? For children with cerebral palsy who have mild impairment and are able to walk (Level I/II), there is a need to increase the amount of their physical activity. Specifically there is a need to increase the amount and frequency of their moderate to vigorous activity. Since cost was one of the main barriers preventing these children from performing more regular activities, then exploring the options for reducing the cost of activities which could be performed more frequently, ie, gym membership and local physical activity programs such as those run by the YMCA, is warranted. Similarly, there is a need to implement more physiotherapy- led activity programs for children with cerebral palsy in their communities. Promoting physical activity in all populations is a health priority and for children with cerebral palsy this is an opportunity for activity which is yet to be developed. Furthermore, initiating programs which can be externally monitored for support and progression of activities will most likely increase the motivation of children to perform home-based activities.

\section{CONCLUSION}

In physiotherapy applications of the children with $\mathrm{CP}$, use the terminology "treatment" only is insufficient. Instead of this terminology, it is more accurate to use "management". Because, physiotherapy of children with CP not only includes the treatment of the motor problems, but also takes all the requirements of the child and a good family-child relation into consideration. During the applications, existing problems of the child should be taught as a whole of motor, cognitive, sensorial, emotional, social areas. It is very important to determine the realistic goals. The rehabilitation team should determine the short and long term targets according to the existing condition of the child and the team should control the targets in certain periods whether if they can reach those targets. If an unreal target is noticed, providing a correct connection between the condition of the child and the target and planning the strategies to reach the targets are the main principles. During the applications, planning all day, working with the family, integration to daily living activities, helping to the child, increasing the quality of life are important. Determination of the main targets during the treatment, taking the child's personal characteristics into consideration, providing the usage of the functional abilities of child during playing were the other important main points.

Overall, the evidence for physiotherapy intervention for children with cerebral palsy is limited and there is clearly a need for improved quality in research. However, research into cerebral palsy is challenging, not only because of severity of impairments and activity limitations, but also the research is undertaken while growth and development are occurring. High quality 
research involving children with cerebral palsy requires commitment and adherence by not only the child, but also the family, and at times the child's local community.

Generally, children with cerebral palsy just want to engage in activities alongside their typicallydeveloping peers. The ongoing challenge for physiotherapy intervention for these children is to find the best intervention which will enhance their level of activity and community participation. For those children with more severe motor impairment, this challenge is always more difficult. However, for children who are ambulant and capable of physical activity, it is important that they are given the opportunity to engage in regular activity which is performed at a moderate to vigorous level. Furthermore, overcoming the barriers which are preventing physical activity means that there is a greater chance that children with cerebral palsy will have a more active lifestyle.

\section{Conflicts of interest: None}

\section{REFERENCES}

[1]. Singhi PD, Ray M, Suri G. Clinical spectrum of cerebral palsy in north India an analysis of 1000 cases. J Trop Pediaty 2002; 48: 162-166.

[2]. Molnar GE. A developmental perspective for the rehabilitation of children with physical disability. Pediatric Ann 1988; 17: 766-776.

[3]. Palisano RJ, Chiarello LA, Orlin M, Oeffinger D, Polansky M, Maggs J, Bagley A, Gorton G. Determinants of intensity of participation in leisure and recreational activities by children with cerebral palsy. Developmental Medicine and Child Neurology 2010; 53: 142-149.

[4]. Hoare BJ, Imms C, Carey L, Wasiak J. Constraintinduced movement therapy in the treatment of the upper limb in children with cerebral palsy: a Cochrane systematic review. Clinical Rehabilitation 2007; 21: 675-685.

[5]. Maclennan A. A temaplate for defining a casual relation between acute intarpatum events and cerebral palsy. interanational consensus statement. BMJ 1999; 319: 1054-1059.

[6]. Boyd RN, Morris ME, Graham HK. Management of upper limb dysfunction in children with cerebral palsy: a systematic review. European J Neurology 2001; 8(Suppl 5): 150-166.

[7]. Palisano RJ, Rosenbaum PL, Walter S, et al. Developmental and reliability of a system to classify gross motor function in child with cerebral palsy. Dev Med Child Neurol 1997; 39: 214-223.

[8]. Rosen MG, Dickinson JC. The incidence of cerebral palsy. AM J obstest Gynecol 1992; 167: 417-423.
[9]. Nelson KB. Can we prevent cerebral palsy?. N eugl J med 2003; 349: 179.

[10]. Kuban KCK, Levilon A. Cerebral palsy. N Eng L J MED 1994; 33: 188-195.

[11].Martin, Kessler: Neurological interventions for physical therapy $2^{\text {nd }}$ edition: 124-126.

[12]. Thelan E, Treadmill stepping in seven month old infants child. Dev 1986; 57: 1498-1506.

[13]. Menkes JH, Sarnat HB. Periutal asphyxia and trauma. In menkesjh, Sarnat HB, edn. Child neurology. Lppinoh Williams and Wilkins 2000; 427-436.

[14]. Wu YW, Colford JMJR. Choioamnionitis as a risk factor for cerebral palsy. A meta analysis JAMA 2000; 284: 1417-1424.

[15]. Walt JM, Robertson CMT, Grace MGA. Early prognosis for ambulation of neonatal intensive care survivors with cerebral palsy. Dev Med Child Neurol 1989; 31: 766-773.

[16]. Boyd RN, Morris ME, Graham HK. Management of upper limb dysfunction in children with cerebral palsy: a systematic review. European J Neurology 2001; 8(Suppl 5): 150-166.

[17]. Blackmore AM, Boettcher-Hunt E, Jordan M, Chan MDY. A systematic review of the effects of casting in children with cerebral palsy: an evidence report of the AACPDM. Dev Med Child Neurol 2007; 49: 781790.

[18]. Hoare BJ, Imms C, Carey L, Wasiak J. Constraintinduced movement therapy in the treatment of the upper limb in children with cerebral palsy: a Cochrane systematic review. Clinical Rehabilitation 2007; 21: 675-685.

[19]. Kerr C, McDowell B, McDonough S. Electrical stimulation in cerebral palsy: a review of effects on strength and motor function. Dev Med Child Neurol 2004; 46: 205-213.

[20]. Ashwal S, Russman BS, Blasco PA, et al. Diagnostic assessment of the child with cerebral palsy: Report of the quality standards subcommittee of the American academy of neurology and the practice committee of the child neurology society.

[21]. Stanger M. Rehabilitation approach for children with cerebral palsy: overview. J Child Neurol 2003; 18: $579-588$.

[22]. Willis JK, Maillo A, Riujl A. et al. Forced use treatment of childhood hemiparesis. Pediatric 2002; 110: 94-96.

[23]. Alpert BS, Flood NL, Strong WB, Dover EV, DuRant RH, Martin AM, Booker DL. Responses to ergometer exercise in a healthy biracial population of children. J Pediatrics 1982; 101: 538-545.

[24]. Mockford M, Caulton JM. Systematic review of progressive strength training in children and adolescents with cerebral palsy who are ambulatory. Pediatric Physical Therapy 2008; 20: 318-333.

[25]. Palisano RJ, Chiarello LA, Orlin M, Oeffinger D, Polansky M, Maggs J, Bagley A, Gorton G. Determinants of intensity of participation in leisure and recreational activities by children with cerebral palsy. Dev Med Child Neurol 2010; 53: 142-149. 
[26]. Clanchy KM, Tweedy SM, Boyd RN, Trost SG. Validity of accelerometry in ambulatory children and adolescents with cerebral palsy. European J Applied Physiology 2011; 12: 2951-2959.

[27]. Dood KJ. Systemic review of the effectiveness of strength training program for people with cerebral palsy. Pediatric Neurol 2004; 11: 60-77.

[28]. Ade-Hall RA, Moore AP. Botulinum toxin type A in the treatment of lower limb spasticity in cerebral palsy. Cochrane Database of Systematic Reviews 2000; 2: CD001408.
[29]. Thompson G, Hrubin K, Bilenker RM. Comprehensive management of cerebral palsy New York. NY. Grune and Stratton. 1983 and Scherzer A. tscharnuter: early diagnosis and therapy in cerebral palsy. AMJ Dis child 1989; 143: 552-555.

[30].Vargos SC, Amilli G. A meta analysis of Research on Sensory Integritive treatment Amjoccr therapy. 1999; 53: 189-198.

How to cite this article:

Raj Sharma, Jyoti Sharma, Vikas Bharadwaj. EVIDENCE BASED REVIEW OF PHYSIOTHERAPY MANAGEMENT OF CEREBRAL PALSY PATIENTS. Int J Physiother Res 2018;6(5):2864-2881. DOI: 10.16965/ijpr.2018.166 